\title{
Application of Femtosecond-Laser Induced Nanostructures in Optical Memory
}

\author{
Yasuhiko Shimotsuma ${ }^{1, *}$, Masaaki Sakakura ${ }^{1}$, Kiyotaka Miura ${ }^{2}$, Jiarong Qiü ${ }^{3}$, \\ Peter G. Kazansky ${ }^{4}$, Koji Fujita ${ }^{2}$, and Kazuyuki Hirao $0^{1,2}$ \\ ${ }^{1}$ Fukui Institute for Fundamental Chemistry, Kyoto University, Nishihiraki-cho 34-4, Takano, Sakyo-ku, \\ Kyoto 606-8103, Japan \\ ${ }^{2}$ Department of Material Chemistry, Graduate School of Engineering, Kyoto University, Nishikyo-ku, \\ Kyoto 615-8510, Japan \\ ${ }^{3}$ Department of Materials Science, Zhejiang University, Hangzhou 310027, China \\ ${ }^{4}$ Optoelectronics Research Centre, University of Southampton, Southampton S017 1BJ, UK
}

\begin{abstract}
The femtosecond laser induced micro- and nanostructures for the application to the threedimensional optical data storage are investigated. We have observed the increase of refractive index due to local densification and atomic defect generation, and demonstrated the real time observation of photothermal effect after the femtosecond laser irradiation inside a glass by the transient lens ( $T r L)$ method. The TrL signal showed a damped oscillation with about an 800 ps period. The essential feature of the oscillation can be reproduced by the pressure wave creation and propagation to the outward direction from the irradiated region. The simulation based on elastodynamics has shown that a large thermoelastic stress is relaxed by the generation of the pressure wave. In the case of soda-lime glass, the velocity of the pressure wave is almost same as the longitudinal sound velocity at room temperature $(5.8 \mu \mathrm{m} / \mathrm{ns})$. We have also observed the localized photo-reduction of $\mathrm{Sm}^{3+}$ to $\mathrm{Sm}^{2+}$ inside a transparent and colorless $\mathrm{Sm}^{3+}$-doped borate glass. Photoluminescence spectra showed that some the $\mathrm{Sm}^{3+}$ ions in the focal spot within the glass sample were reduced to $\mathrm{Sm}^{2+}$ ions after femtosecond laser irradiation. A photo-reduction bit of $200 \mathrm{~nm}$ in three-dimensions can be recorded with a femtosecond laser and readout clearly by detecting the fluorescence excited by $\operatorname{Ar}^{+}$laser $(\lambda=488 \mathrm{~nm})$. A photo-reduction bit can be also erased by photo-oxidation with a $\mathrm{cw}$ $\operatorname{Ar}^{+}$laser $(\lambda=514.5 \mathrm{~nm})$. Since photo-reduction bits can be spaced $150 \mathrm{~nm}$ apart in a layer within glass, a memory capacity of as high as 1 Tbit can be achieved in a glass piece with dimensions of $10 \mathrm{~mm} \times 10 \mathrm{~mm} \times 1 \mathrm{~mm}$. We have also demonstrated the first observation of the polarizationdependent periodic nanostructure formation by the interference between femtosecond laser light and electron acoustic waves. The observed nanostructures are the smallest embedded structures ever created by light. The period of self-organized nanostructures can be controlled from $\sim 140$ to $320 \mathrm{~nm}$ by the pulse energy and the number of irradiated pulses. Furthermore, we have also observed the self-assembled sub-wavelength periodic structures created in silica glass by femtosecond pulses on the plane of the propagation of light.
\end{abstract}

Keywords: Ultrashort Pulse Laser, Three-Dimensional Optical Memory, Refractive Index, Rare Earth Doped Glass, Interference, Nanostructure, Plasma.

\section{CONTENTS}

1. Introduction $\ldots \ldots \ldots \ldots \ldots \ldots \ldots \ldots \ldots \ldots \ldots \ldots$

2. Localized Microstructures Formation by Femtosecond Laser Irradiation

3. Mechanisms of Localized Refractive Index Change Induced by Femtosecond Laser Irradiation . . . . . . . . . . . . . . 4

4. Space-Selective Valence State Manipulation of Active Ions. . . 6

5. Single Femtosecond Laser Beam Induced Polarization-Dependent Periodic Nanostructures

*Author to whom correspondence should be addressed.

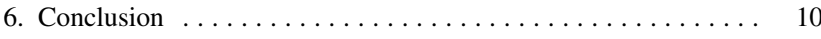

Acknowledgments ........................ 10

References and Notes .................. 11

\section{INTRODUCTION}

The optical storage density has shown rapid progress in the information technologies. There are two methods to increase the storage density;

(1) reducing the size of a record bit,

(2) making a multivalued or multilayered bit structure. 


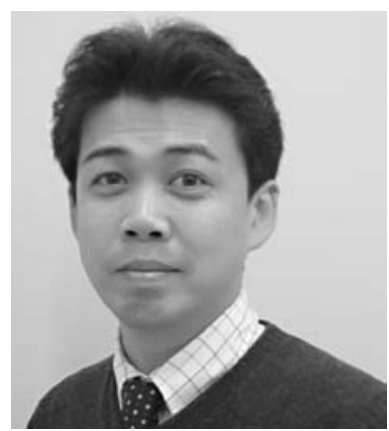

Koji Fujita was born in Osaka, Japan, in 1971. He is an associate professor of Department of Material Chemistry, Kyoto University. He graduated from Division of Industrial Chemistry, Kyoto University in 1994, and received his M.S. degree in material chemistry in 1996 under the direction of Prof. Naohiro Soga (who is currently an emeritus professor of Kyoto University), and his Ph.D. degree from Kyoto University in 1999 under the supervision of Prof. Kazuyuki Hirao. After working at Kyoto University as a postdoctorial fellow of Japan Society for the Promotion of Science (JSPS), he joined Department of Materials Chemistry, Kyoto University in 1999, as a research associate. In 2005, he was appointed as an associate professor. He was awarded the Ceramic Society of Japan (CerSJ) Award for Young Ceramists in 2002, and the Chemical Society of Japan (CSJ) Award for Young Chemists in 2006. His current research interests lie in the development of new oxide-based glasses and/or ceramics for photonics and spinelectronics.

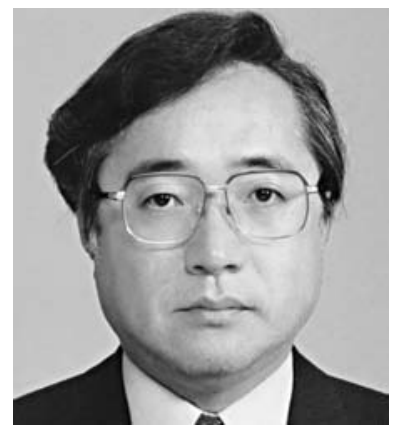

Kazuyuki Hirao received B.Sc., M.Sc., and Ph.D. degrees in Kyoto University in 1974, 1976, and 1979, respectively. He is currently a professor of Kyoto University, and holds the director of Photon Craft Project, ICORP, JST and the director of Nanoglass Project, MITI. He was postdoctoral fellow at Rensselaer Polytech. Inst. from 1985 to 1986, and held the director of Hirao Active Glass Project, ERATO, JST from 1994 to 1999. He received New Chemistry Society Prize for 1990, Gottardi International Prize for 1990, Ceramic Society of Japan Prize for 1998, Chemistry Society of Japan Prize for 2000, Morey International Prize for 2002, American Ceramic Society Fellow awarded in 2002, the Minister of Economy, Trade, and Industry in Japan Prize for 2004. He has published more than 411 original and 177 review papers and books in his field. His work covers a broad range of applications including creations and analysis of photo-induced structures in glasses, functional devices using active glasses, structure and properties of glasses containing transition and rare earth elements and amorphous materials made by unconventional method, phase separation of inorganic-organic polymer systems, and molecular dynamics simulation of glass. He is now a regional editor of Journal of Non-Crystalline Solids.

In the first case, the many technologies concerning the shortening of the wavelength of a light source have been innovated due to the diffraction limit of light. However, it is well known that the limit of the high densification of an optical data storage by the optical diffraction is $20 \sim 30 \mathrm{Gbit} / \mathrm{inch}^{2}$. Furthermore, the information storage density is estimated to reach into $1 \mathrm{Tbit} / \mathrm{inch}^{2}$ in the next decade. In fact, the processing technology with a bit size of about $25 \mathrm{~nm}$ is required. On the other hand, in the latter case, increase of density can be realized by writing the bits in a three dimension space. ${ }^{1}$ Misawa et al. observed the picosecond-pulsed YAG laser-induced refractive-index-changed microspot inside silicate glass in 1995, and suggested that the observed phenomenon can be used for three-dimensional optical memory. ${ }^{2,3} \mathrm{We}$ started the systematic investigations on the femtosecond laser induced microstructures inside transparent materials and applications in micro-optics at the end of 1994. In the early stages of research on micromachining using ultrashort laser pulses, Mazur et al. demonstrated a method for high-density three-dimensional optical data storage using femtosecond laser pulses in $1996 .{ }^{4}$ They recorded submicron-diameter bits consisting of a void surrounded by a densified region by focusing a femtosecond laser inside fused silica. When femtosecond laser pulses are tightly focused inside a glass by a focusing lens, the strength of the electric field at the focal point of the laser beam can reach $\sim 10 \mathrm{TW} / \mathrm{cm}^{2}$, which is sufficient for inducing various nonlinear physicochemical reactions in materials. The photo-induced reactions are expected to occur only near the focused part of the laser beam due to multiphoton processes. Besides, the nature of the interaction between femtosecond laser pulses and matter makes it possible to overcome the diffraction limit of light. ${ }^{5}$ In the past several couple of years, a lot of research efforts have been devoted to the field of the three-dimensional microscopic modifications to transparent materials by using femtosecond laser. Promising applications have been demonstrated for the formation of a three-dimensional optical memory, ${ }^{4,6-9}$, and also fabrication of optical waveguides, ${ }^{10,11}$ couplers ${ }^{12,13}$ and photonic crystals. ${ }^{14}$ In this paper, we review our recent investigations on the femtosecond laser-induced nanostructures for the optical data storage applications. The mechanisms and possible applications of the observed phenomena are also discussed.

\section{LOCALIZED MICROSTRUCTURES FORMATION BY FEMTOSECOND LASER IRRADIATION}

Figure 1 shows various localized structural changes can be induced inside a glass sample by focusing a femtosecond laser operating at a non-resonant wavelength. There are 
(a)
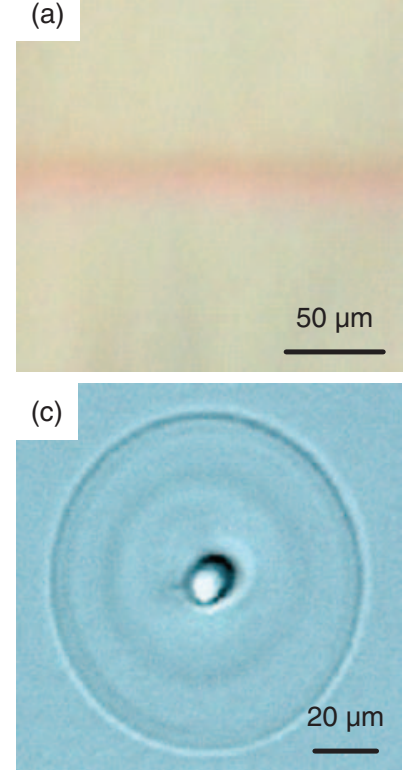

(b)

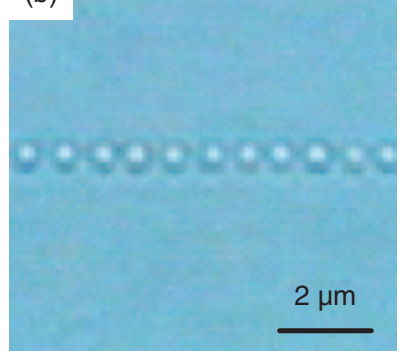

(d)

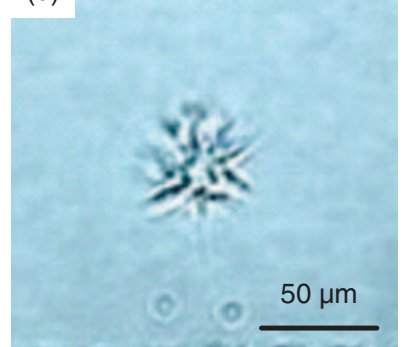

Fig. 1. Various induced localized microstructures by the infrared femtosecond laser pulses.

four examples of induced structural change:

(a) coloration due to the color center formation and valence state change of active ions such as rare-earth and transition metal ions,

(b) refractive index change due to local densification and atomic defect generation,

(c) micro-void formation due to localized re-melting and shock wave propagation, and

(d) micro-crack formation due to destructive breakdown or other phenomena.

The induced microstructures between (a) and (c) are directly applicable to the three-dimensional optical memory. A basic explanation of the various femtosecond laserinduced phenomena is as follows. Usually glass has no absorption at $800 \mathrm{~nm}$ (the writing wavelength of the Ti: sapphire laser). Linear absorption of the laser radiation does not occur when the glass is irradiated by the laser beam. This is because the energy gap between the valence and conduction bands is larger than the energy of the single photon. Thus a single photon does not contain sufficient energy to excite an electron at the ground state. At sufficiently high laser intensities, however, an electron can simultaneously absorb the energy from the multiple photons to exceed the band gap. This nonlinear process is a highly intensity-dependent process, with the rate $P(I)=$ $\sigma_{k} I^{k}$, where $\sigma_{k}$ is the multiphoton absorption coefficient for $k$-photon absorption (multiphoton absorption). Once an electron is promoted to the conduction band, it serves as a seed to a process called avalanche ionization. ${ }^{15}$ Seeds can also arise from the other processes such as electron tunneling and thermal excitation from impurity states. An electron in the conduction band can absorb sufficient energy $n h \nu>E_{\mathrm{g}}$, where $n$ is the number of photons absorbed

sequentially and $E_{\mathrm{g}}$ is the bandgap energy, it can then use the excess energy to ionize another electron via direct collision, also known as impact ionization. The resulting two electrons in the conduction band can then continue the process of the linear absorption and impact ionization to achieve an exponential growth of the free electrons. Such avalanche ionization produces highly absorptive and dense plasma, facilitating the transfer of energy from the laser to the glass. The resulting melting, material displacement due to the plasma expansion, and chemical restructuring resulted into various induced structures.

\section{MECHANISMS OF LOCALIZED REFRACTIVE INDEX CHANGE INDUCED BY FEMTOSECOND LASER IRRADIATION}

Figure 2 shows a result of the read out of the data dots stored inside the $\mathrm{SiO}_{2}$ glass by the femtosecond laser pulses that emits $800 \mathrm{~nm}, 120 \mathrm{fs}$, and $200 \mathrm{kHz}$. A remarkable refractive index increase was observed at the center of the writing region. ${ }^{10}$ The laser fluence was about $1.2 \times$ $10^{2} \mathrm{~J} / \mathrm{cm}^{2}$ and a $100 \mathrm{X}$ lens with an NA of 0.95 was used to focus the writing laser light. The writing time was $1 \mathrm{~s}$ on one dot and the distance between the adjacent dots was $2 \mu \mathrm{m}$. The size of a dot formed under this condition was $1 \mu \mathrm{m}$. The formation of $\mathrm{SiE}^{\prime}$ color center was mainly observed in the laser writing $\mathrm{SiO}_{2}$ glass sample, and also peroxy-radicals and non-bridge oxygen hole centers (NBOHC) were observed (Fig. 3) after the femtosecond laser writing. ${ }^{6}$ Since the glass samples are transparent in $800 \mathrm{~nm}$, we suggest that a multiphoton absorption process occurred in the vicinity of the femtosecond laser pulses focal region.

We previously observed a densification phenomenon in the glass irradiated by the $800 \mathrm{~nm}$ focused femtosecond

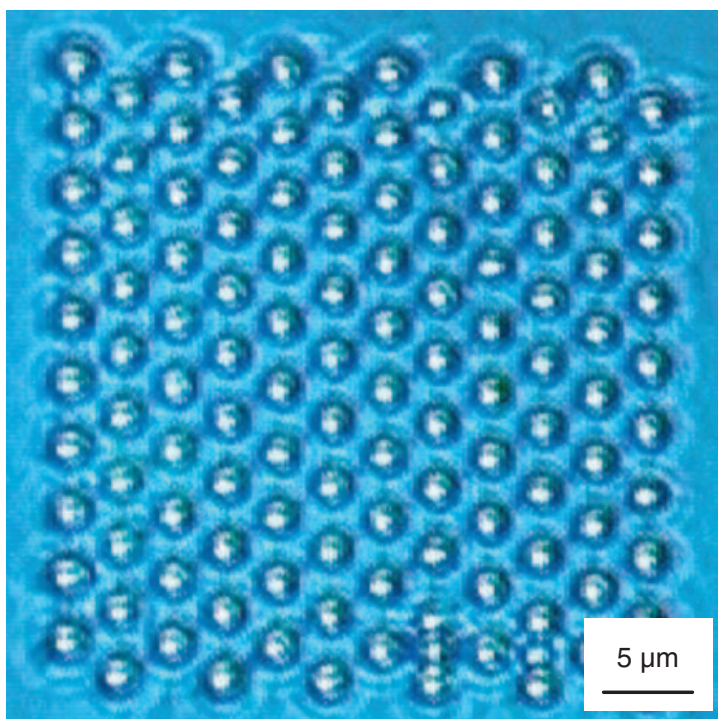

Fig. 2. A result of the read out of the data information stored inside the $\mathrm{SiO}_{2}$ glass. The size of a bit is $1 \mu \mathrm{m}$. 


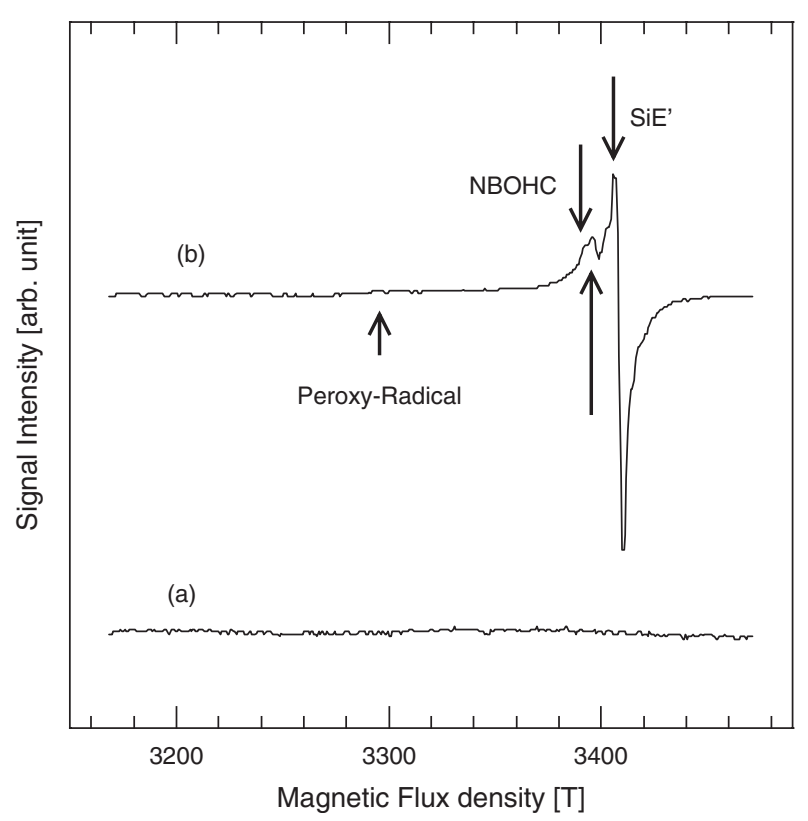

Fig. 3. ESR spectra of the $\mathrm{SiO}_{2}$ glass before (a) and after (b) the femtosecond laser irradiation. Reprinted with permission from [6], J. Qiu et al. Jpn. J. Appl. Phys. 37, 2263 (1998). (C) 1998, Institute of Pure and Applied Physics.

laser pulses from the observation of AFM. ${ }^{11}$ Therefore, we suggest that the formation of defect centers such as $\mathrm{SiE}^{\prime}$, and densification may cause the increase in refractive index at the center of the laser writing dot. In addition, the ultrafast energy deposition may also create high temperatures and pressures inside the glasses. Re-melting and rapid quenching may occur during the laser writing process, resulting in localized densification in the glasses.

For understanding the mechanism of refractive-index change, we observed the initial step of the refractive index change in the small irradiated area inside a bulk glass by pump-probe method with a time resolution of sub-ps. ${ }^{16}$ Details of this method have been described elsewhere. ${ }^{17}$ Figure 4 shows the temporal evolution of the signal intensity, which is called TrL signal, after the femtosecond laser irradiation (laser power: $0.55 \mu \mathrm{J} /$ pulse, pulse width: $500 \mathrm{fs}$ ). This signal intensity is defined as the light intensity at the center of the probe beam. The intensity distributions of the probe beam at various delay time are shown in the inset of Figure 4. The TrL signal suggests that the refractive index changes dramatically after the photoirradiation. In the shorter time range [Fig. 4(a)], the TrL signal intensity decreases immediately, and returns back to the same level before excitation. The time duration of this signal is as short as that of the pump pulse ( $\sim 500 \mathrm{fs})$. Considering the ultrafast response of the signal, the main origin of the signal should be attributed to the electronic response of the optical Kerr effect ${ }^{18}$ and photo-induced plasma, which decays as fast as $1 \mathrm{ps}$ in $\mathrm{SiO}_{2}{ }^{19}$

In a long time range, the TrL signal oscillates with a time period of about 800 ps and the oscillation decays
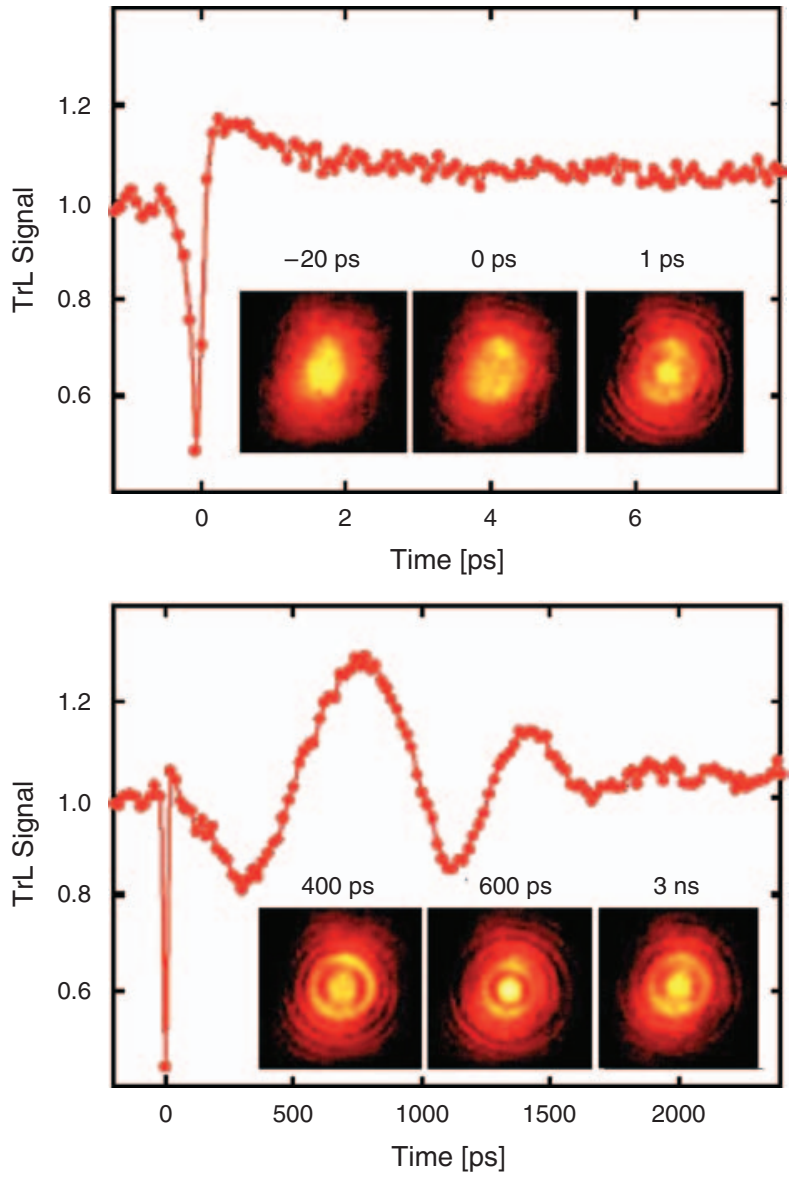

Fig. 4. Observed TrL signals after the irradiation of the femtosecond laser pulse inside a glass (a) in the short time range, (b) in the long time range. Insets show the images of the probe beam detected by the CCD camera at various delay times. Reprinted with permission from [17], M. Sakakura and M. Terazima, Phys. Rev. B 71, 024113 (2005). (C) 2005, The American Physical Society.

in about 2 ns [Fig. 4(b)]. We found that the propagation of the pressure wave can reproduce this oscillating $\operatorname{TrL}$ signal. ${ }^{17}$

In the TrL method, the intensity distribution of the probe beam $\left(I_{\mathrm{SIG}}(s) ; s\right.$ is the radial position from the beam center.) can be calculated by the phase change $(\Delta \phi(r) ; r$ is the radial position from the center of the irradiated region), which corresponds to the refractive index change in the femtosecond laser focal region. Therefore, the refractive index distribution in the laser focal region can be calculated from the intensity distribution of the probe beam (Fig. 4 insets). This calculation is known as phase retrieval method. Figure 5 shows the temporal evolution of the phase distribution $\Delta \phi(r)$ obtained by the phase retrieval calculation. The phase distribution changes immediately after the photo-irradiation (1 ps). We attributed this immediate change to the fast temperature elevation, because the temperature change should induce the refractive index change and it is reported that the energy of the hot electrons produced by the photo-excitation is transferred to the lattice within 1 ps in $\mathrm{SiO}_{2}$. As a result of the 


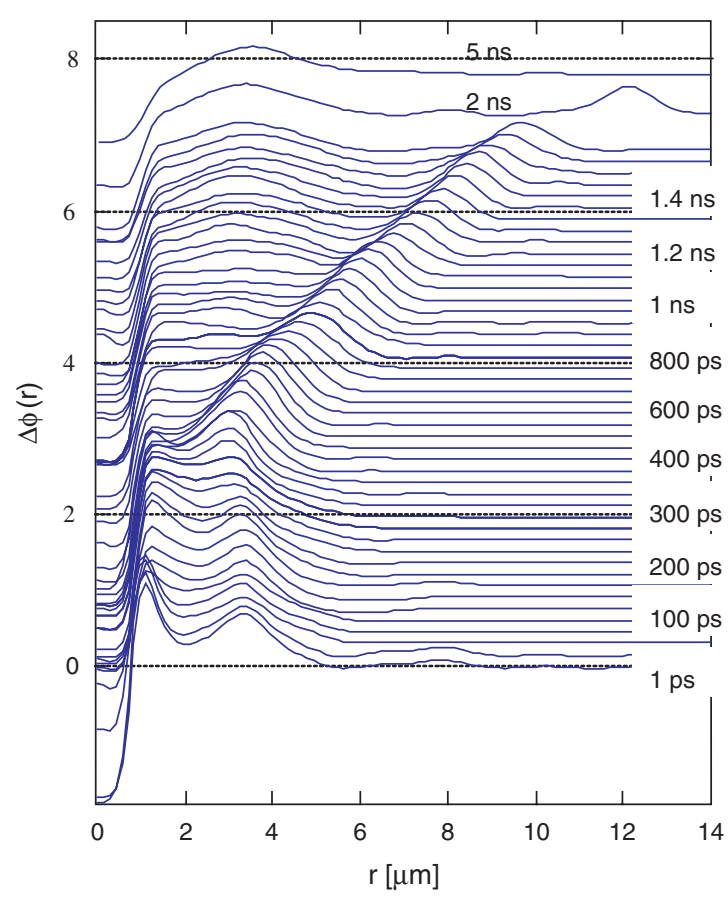

Fig. 5. The temporal evolution of the phase variation function $\Delta \phi(t, r)$ obtained by this phase retrieval calculation of the TrL images. All traces are offset for clarity; the base line $(\Delta \phi=0)$ in each trace is on the same level as the point at $r=12 \mu \mathrm{m}$. Reprinted with permission from [17], M. Sakakura and M. Terazima, Phys. Rev. B 71, 024113 (2005). (C) 2005, The American Physical Society.

fast temperature change, a large thermoelastic stress is confined in the laser focal region at the fast time range, and the large stress should be relaxed in a longer time range. The simulation based on elastodynamics has shown that the confined stress is relaxed by the generation of the pressure wave. Figure 5 shows that the phase around $3.0 \mu \mathrm{m}$ increases between $300 \mathrm{ps}$ and $600 \mathrm{ps}$ and the position of this positive phase peak changes gradually in the outward direction. The generation and propagation of this phase peak correspond to the pressure wave generation and propagation, respectively. After $600 \mathrm{ps}$, the positive peak due to the pressure wave propagates outward with a constant velocity $(5.8 \mu \mathrm{m} / \mathrm{ns})$.

The velocity of the pressure wave is almost same as the longitudinal sound velocity in the soda-lime glass at room temperature $(5.8 \mu \mathrm{m} / \mathrm{ns})$. It suggests that the temperature change does not occur in the surrounding region by the laser irradiation. When the pressure wave escapes away from the probe beam region $(t>2000 \mathrm{ps})$, the temporal change in $\Delta \phi(t, r)$ is very minor as evident by the comparison of $\Delta \phi(r)$ between 2000 ps and 5000 ps in Figure 5. Since the thermal diffusion time is calculated to be much longer ( $>100 \mathrm{~ns}$ ) than the observation time range in this study, the thermal energy still remains in the irradiated region at $5000 \mathrm{ps}$. This is the first real time observation of the temporal evolution of the refractive index distribution at the laser-irradiated region inside a glass induced by the ultrashort laser pulse. We hope that this technique will be a powerful tool to elucidate the ultrafast refractive index increase by the laser pulse.

\section{SPACE-SELECTIVE VALENCE STATE MANIPULATION OF ACTIVE IONS}

Recently, we demonstrated the thee-dimensional optical data storage by using the femtosecond laser-induced valence state change of rare earth ions in glasses. ${ }^{20,21}$ Room-temperature permanent photo-reduction of $\mathrm{Sm}^{3+}$ to $\mathrm{Sm}^{2+}$ was observed in borate and other glass samples. Figure 6 shows photoluminescence spectra obtained by excitation at $488 \mathrm{~nm}$ for a laser-irradiated area (a) and non-irradiated area (b) in the interior of the glass. Comparing (a) to (b) shows that the emission in the $650 \sim$ $775 \mathrm{~nm}$ region differed appreciably. The broad bands observed around 560, 600, and $645 \mathrm{~nm}$ can be attributed to the ${ }^{4} \mathrm{G}_{5 / 2} \rightarrow{ }^{6} \mathrm{H}_{5 / 2,7 / 2,9 / 2}$ transitions, respectively, of the $\mathrm{Sm}^{3+}$ ions. On the other hand, the emissions at 680, 700, and $725 \mathrm{~nm}$ are attributed to the ${ }^{5} \mathrm{D}_{0} \rightarrow{ }^{7} \mathrm{~F}_{0,1,2}$ transitions, respectively, of the $\mathrm{Sm}^{2+}$ ions. This means that laser-irradiated areas (photo-reduced areas) recorded inside glass can be detected only by emissions at 680,700 , or $725 \mathrm{~nm}$. By using the photo-reduction of $\mathrm{Sm}^{3+}$ to $\mathrm{Sm}^{2+}$, alphabetical characters were recorded in the form of submicron size bits in a three-dimensional (layered) manner in a glass sample. Here, one recorded character consisted of 300 to 500 photo-reduction bits recorded with 5000 laser shots per bit. As a recording source we employed a regeneratively amplified $800 \mathrm{~nm}$ Ti: sapphire laser emitting $20 \mathrm{~Hz}$ or $250 \mathrm{kHz}$ mode-locked pulses. We tightly focused femtosecond laser pulses inside the bulk glass to

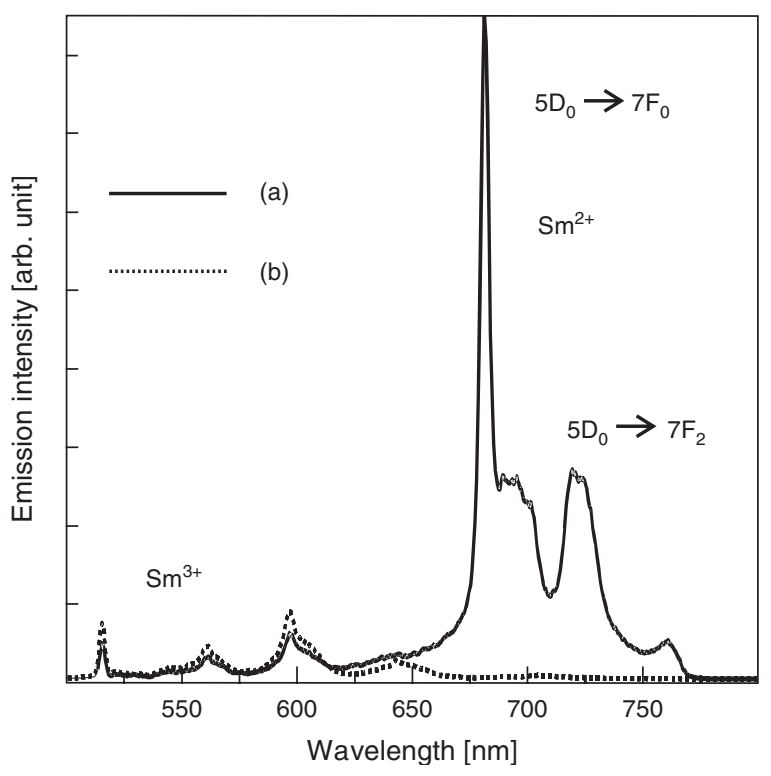

Fig. 6. Photoluminescence spectra obtained by excitation at $488 \mathrm{~nm}$ for a laser-irradiated area (a) and non-irradiated area (b) in the interior of the glass. Reprinted with permission from [21], K. Miura et al., Appl. Phys. Lett. 80, 2263 (2002). (C) 2002, American Institute of Physics. 

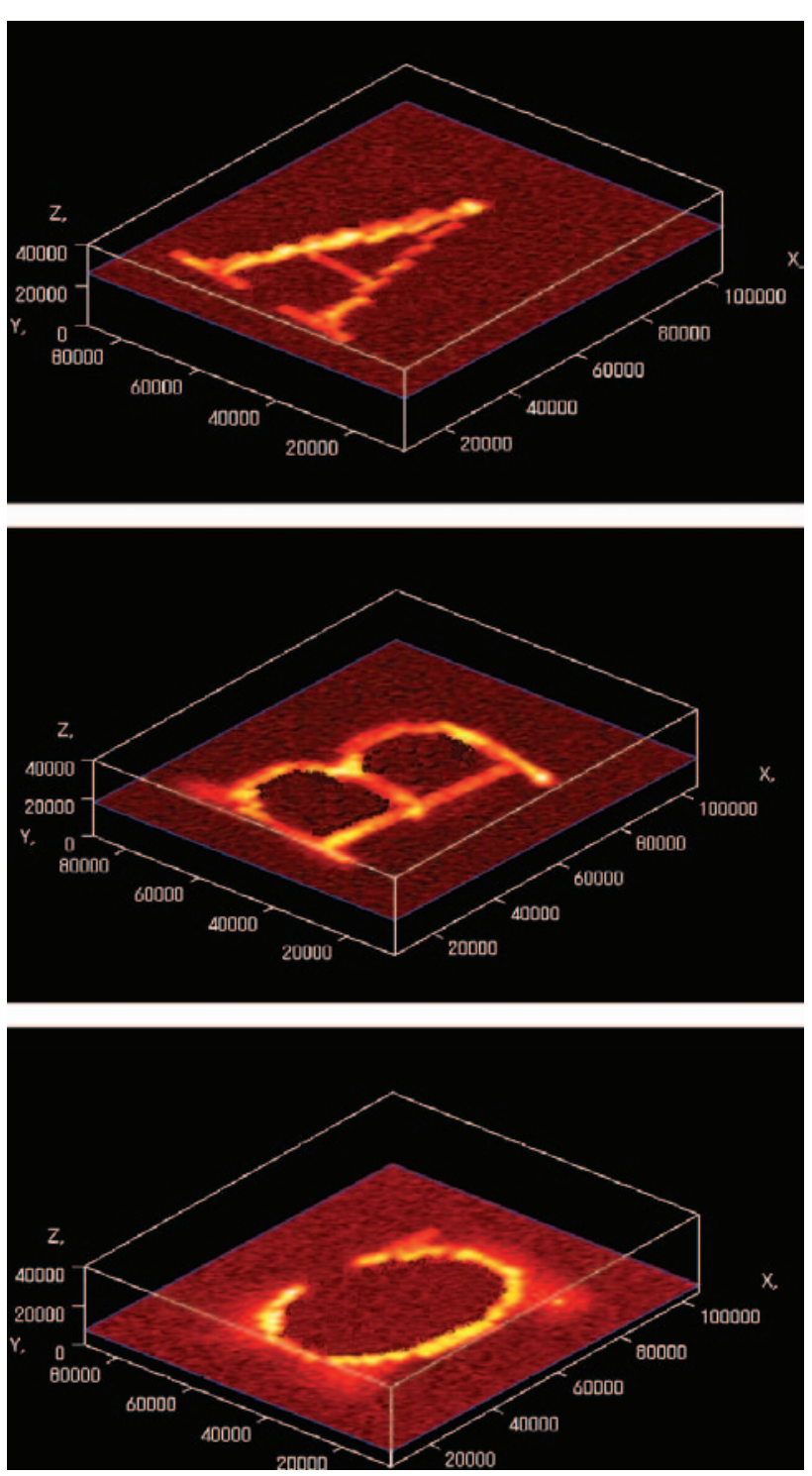

Fig. 7. Photoluminescence images of alphabetical characters recorded on different layers, which were observed by using a $40 \mathrm{X}$ objective lens and the $680 \mathrm{~nm}$ emission from $\mathrm{Sm}^{2+}$ with confocal detection implemented (excitation at $488 \mathrm{~nm}, 1 \mathrm{~mW} \mathrm{Ar}{ }^{+}$laser). Reprinted with permission from [19], K. Miura et al., Appl. Phys. Lett. 80, 2263 (2002). (c) 2002, American Institute of Physics.

locally photo-reduce $\mathrm{Sm}^{3+}$ to $\mathrm{Sm}^{2+}$. The spacing between alphabetical characters was $2 \mu \mathrm{m}$. The bits had a diameter of $400 \mathrm{~nm}$, which was significantly smaller than the focal-beam size and the wavelength of the recording laser. Figure 7 shows photoluminescence images of alphabetical characters recorded on different layers, which were observed by using a $40 \mathrm{X}$ objective lens and the $680 \mathrm{~nm}$ emission from $\mathrm{Sm}^{2+}$ with confocal detection implemented. We confirmed that the spacing of $2 \mu \mathrm{m}$ between alphabetical character planes was sufficient to prevent cross-talk in the photoluminescence images. Although the threedimensional memory bits (photo-reduced areas) were recorded with a femtosecond laser, they could be read with a CW laser at $0.5 \mathrm{~mW}$.
The results demonstrated the possibility of selectively inducing a change of valence state of $\mathrm{Sm}^{3+}$ ions on the micrometer scale inside a glass sample by use of a focused non-resonant femtosecond laser pulses. Whereas a three-dimensional optical memory has approximately $10^{13} \mathrm{bits} / \mathrm{cm}^{3}$ storage density, which means that data information can be stored in the form of a change in refractive index in a spot, optical memory using a valence-state change of rare earth ions in a spot may have the same storage density and may allow one to read out data in the form of luminescence, thus providing the advantage of a high signal-to-noise ratio. Therefore, the present technique will be useful in the fabrication of three-dimensional optical memory devices with high storage density. Moreover, femtosecond laser photo-reduced $\mathrm{Sm}^{3+}$-doped glasses exhibited a photochemical spectral hole burning memory property. ${ }^{22}$ The microspot induced by the focused femtosecond laser inside a glass sample can be further used to store data information via the irradiation of laser light with different wavelengths. As a result, the data information
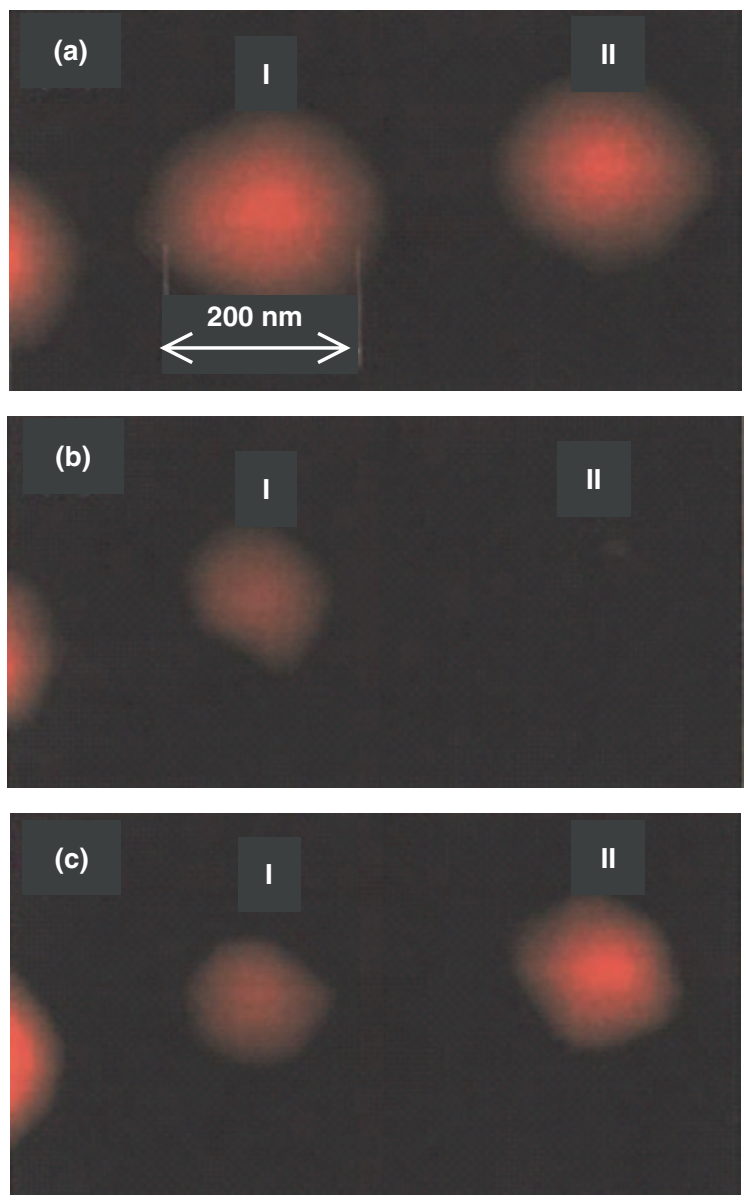

Fig. 8. Example of three-dimensional rewriteable optical memory using photo-reduction of $\mathrm{Sm}^{3+}$ by irradiation of femtosecond laser and an $\mathrm{Ar}^{+}$ laser at $514 \mathrm{~nm}$. (a) writing bit II by femtosecond laser, (b) erasing bit II by $\mathrm{Ar}^{+}$laser, and (c) rewriting bit II by femtosecond laser, respectively. Reprinted with permission from [21], K. Miura et al., Appl. Phys. Lett. 80, 2263 (2002). (C) 2002, American Institute of Physics. 
can be read out in the form of spectral holes. $\mathrm{Sm}^{2+}$-doped glasses could become an ultimate optical memory device with an ultrahigh storage density.

We also observed that photo-reduced $\left(\mathrm{Sm}^{3+} \rightarrow \mathrm{Sm}^{2+}\right)$ submicron bit written by using the femtosecond laser can be erased by irradiation with an $\mathrm{Ar}^{+}$laser at $514.5 \mathrm{~nm}$ (Fig. 8). ${ }^{21}$ This result indicated the possibility of achieving a three-dimensional optical memory with rewrite capability.

\section{SINGLE FEMTOSECOND LASER BEAM INDUCED POLARIZATION-DEPENDENT PERIODIC NANOSTRUCTURES}

Interaction of radiation with matter attracts considerable interest in many areas of science. In particular, since the discovery of the laser, many studies have been carried out on the interaction of intense laser radiation with glass, which is a key material in modern optical technology. It is well known that the scattering of polarized light in the plane of light polarization in an isotropic medium, such as glass, is always weaker compared to the orthogonal plane, since a dipole does not radiate in the direction of its axis. In 1999, however, we observed a new phenomenon in Gedoped silica glass pumped by intense laser radiation-the scattering of light, in particular luminescence, which peaks in the plane of light polarization (anomalous anisotropic light scattering). ${ }^{23}$ The phenomenon is interpreted in terms of the angular distribution of photoelectrons in isotropic solid state materials.

When the pump $(10 \mathrm{~mW}$ average power, $0.4 \mathrm{MW}$ peak power, $2.5 \times 10^{12} \mathrm{~W} / \mathrm{cm}^{2}$ intensity in the focus of a beam) was focused slightly $(\sim 50 \mu \mathrm{m})$ above the surface of the sample the shape of the spot of the blue luminescence imaged via the microscope and CCD camera was circular. Unexpectedly, it has been discovered that when the pump was focused inside the sample the spatial isotropy of the blue luminescence can be broken: The luminescence scattering increases along the direction of the pump polarization, while the circular shape of the pump beam remains unchanged. If we rotate the direction of the pump polarization by using a half-wave plate, the elongated pattern of the blue luminescence follows this rotation. The observed phenomenon represents the first evidence of anisotropic light scattering, which peaks in the plane of light polarization in isotropic media. The phenomenon was considered to be due to the angular distribution of photoelectrons in isotropic solid materials.

Later, we observed memorized polarization-dependent light scattering phenomenon in a $\mathrm{Eu}^{2+}$-doped fluoroaluminate glass sample, and then various glasses and crystals. ${ }^{24}$ In the case of the $\mathrm{Eu}^{2+}$-doped glass, a regeneratively amplified $800 \mathrm{~nm}$ Ti: sapphire laser emitting 120 fs, $200 \mathrm{kHz}$, mode-locked pulses was used in our experiments. The laser beam (in the Gaussian mode at an average power of $100 \mathrm{~mW}$ ) was focused using a $10 \mathrm{X}$ objective lens with a numerical aperture of 0.30 towards the interior of the glass sample of $4 \mathrm{~mm}$ thickness with an XYZ stage. The pump spot size in the focus of the beam was $8 \mu \mathrm{m}$, simultaneously; the irradiated spot was imaged in the visible spectral range via an optical microscope using a color CCD (charge-coupled device) camera. When the laser beam $\left(0.5 \mu \mathrm{J}\right.$ energy, $0.84 \times 10^{12} \mathrm{~W} / \mathrm{cm}^{2}$ intensity in the focus of the beam) was focused slightly inside the sample, we observed a circular spot of blue luminescence via the observation of the optical microscope and CCD camera at the beginning. Then, a blue luminescence was observed along the direction of the pump polarization and the length of the blue luminescence pattern increased with the passage of time. The pattern became stable after about five minutes. Once formed, the anisotropic blue luminescence pattern appeared instantaneously every time the laser beam's path to the sample was blocked and then unblocked. When we changed the direction of the light polarization and irradiated the same location, we observed an anisotropic blue luminescence pattern identical to the original one at the beginning, but then observed a new pattern along the new direction of the light polarization while the original blue luminescence pattern disappeared gradually with the passage of time. We suggest that the polarization-dependent permanent microstructure was induced during the intense laser irradiation. Recently, we observed the single femtosecond laser beam-induced polarization-dependent nanostructures..$^{25}$

In our experiments we used commercially available synthetic silica glass, ED-H (Tosoh Quartz Corp., brands of wet silica with $\mathrm{OH} \sim 50 \mathrm{ppm}$ ) of $10 \mathrm{~mm} \times 10 \mathrm{~mm} \times$ $2 \mathrm{~mm}$ size. The laser radiation in Gaussian mode produced by regenerative amplified mode-locked Ti: Sapphire laser (150 fs pulse duration, $200 \mathrm{kHz}$ repetition rate) operating at a wavelength of $800 \mathrm{~nm}$ was focused via $100 \mathrm{X}$ (NA = 0.95) microscope objective into the silica glass samples placed on the XYZ piezo-translation stage. The beam was focused at $\sim 100 \mu \mathrm{m}$ below the surface and the beam waist diameter was estimated to be $\sim 1 \mu \mathrm{m}$. The laser writing parameters were controlled by an electronic shutter, a variable neutral density filter and a half-wave plate placed in an optical path of the laser beam. After laser irradiation the sample was polished to the depth of the beam waist location. The surface of the polished sample was analyzed by scanning electron microscope and Auger electron spectroscopy. Secondary electron (SE) images and backscattering electron (BE) images of the same surface were compared (Fig. 9).

It is well known that the SE image reveals the surface morphology of a sample, while the BE image is sensitive to the atomic weight of the elements or the density of material constituting the observation surface. The SE images of the polished silica sample indicate that the morphology of an irradiated sample in the examined crosssection almost does not change, namely, a void does not 


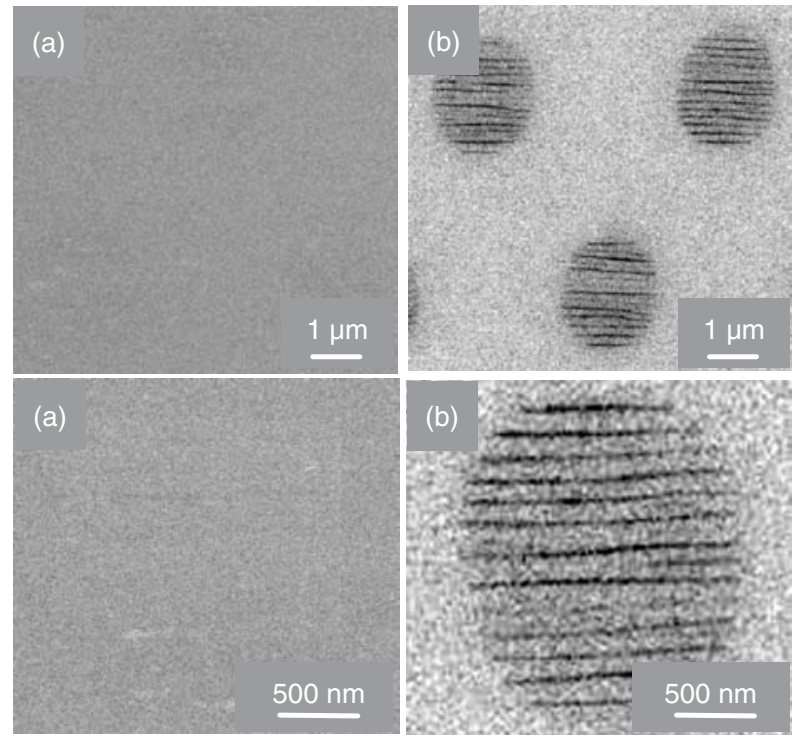

Fig. 9. (a) Secondary electron images of silica glass surface polished close to the depth of focal spot. (b) Light "fingerprints": Backscattering electron images of the same surface. The magnification of the upper and lower images is $10000 \mathrm{X}$ and $30000 \mathrm{X}$, respectively. Reprinted with permission from [25], Y. Shimotsuma et al., Phys. Rev. Lett. 91, 247405 (2003). (C) 2003, The American Physical Society.

exist. On the other hand, the BE images reveal a periodic structure of stripe-like dark regions with low density of material and of $\sim 20 \mathrm{~nm}$ width which are aligned perpendicular to the writing laser polarization direction. Horizontal striation, slightly visible in the SE images [Fig. 9(a)], could be explained by a weak surface relief created in the polishing process due to the density variations in the grating. We speculated, based on the fact that the elements constituting the sample are silicon and oxygen (average molecular weight of $\mathrm{SiO}_{2}$ glass $\sim 60.1$ ), that the oxygen defects were formed in the regions corresponding to dark domains of the $\mathrm{BE}$ image, which reduce the average molecular weight in these regions $\left(\mathrm{SiO}_{2-x} \sim 60.1-16 x\right)$.

To test this suggestion we carried out Auger spectra mapping of silicon and oxygen on the same surface with $10 \mathrm{~nm}$ spatial resolution. The Auger signal of the oxygen in the regions corresponding to dark domains in the $\mathrm{BE}$ image is lower compared to other regions, indicating low oxygen concentration in these domains. Furthermore, the intensity of the oxygen signal is stronger in the regions between the dark domains of the BE image. On the other hand, the intensity of the silicon signal is the same in the whole imaged region. These results indicate that the periodic structure observed in the $\mathrm{BE}$ image consists of periodically distributed oxygen-deficient regions $\left(\mathrm{SiO}_{2-x}\right)$. The Auger signal intensity is proportional to the concentration of element constituting the surface, which gives an estimate to the value $x \sim 0.4$.

We have also observed the birefringence phenomenon at the femtosecond laser irradiated spot due to the oxygen defect domains are formed in the shape of periodic nanostructure. Modulation size of this periodic nanostructure $(\sim 140 \mathrm{~nm})$ is smaller than the light wavelength. So we evaluated the refractive index change, ${ }^{26}$ then the index change in the oxygen defect domain is about minus $0.1 .^{27}$ We observed the decrease of the grating period with an increase of the exposure time. The grating periods were about $240 \mathrm{~nm}, 180 \mathrm{~nm}$, and $140 \mathrm{~nm}$ for the number of light pulses of $5 \times 10^{4}, 20 \times 10^{4}$, and $80 \times 10^{4}$, respectively and for the pulse energy of $1 \mu \mathrm{J}$, corresponding to intensity of $2 \times 10^{14} \mathrm{~W} / \mathrm{cm}^{2}$. This indicated a logarithmic dependence of the grating period $\Lambda$ on the number of light pulses. The dependence of the observed periodic nanostructures on pulse energy for a fixed exposure time was also investigated and an increase of the period with the pulse energy was observed. Grating periods of $180 \mathrm{~nm}$, $240 \mathrm{~nm}$, and $320 \mathrm{~nm}$ were measured at pulse energies of $1 \mu \mathrm{J}, 2 \mu \mathrm{J}$, and $2.8 \mu \mathrm{J}$, respectively and for the number of light pulses of $20 \times 10^{4}$. We have also observed nanovoid grating is formed in other material such as elastooptical crystals represented by tellurium dioxide crystal $\left(\mathrm{TeO}_{2}\right.$ tetragonal crystal, space group: $\left.\mathrm{P}_{1} 2_{1} 2\right){ }^{28}$ Due to the low melting point about $730^{\circ} \mathrm{C}$, and the low binding energy of $\mathrm{Te}-\mathrm{O}$ bond, (that is about $2.5 \mathrm{eV}$ lower than $\mathrm{Si}-\mathrm{O}$ bond), nano-void grating is formed and aligned in the plane perpendicular to the laser polarization direction.

We also observed the cross-section of the polarizationdependent periodic nanostructure (Fig. 10). The single femtosecond laser beam with the different linear polarization directions was irradiated inside a fused silica at a $100 \mu \mathrm{m}$ under the sample surface and scanned the focal spot in the X-Y plane perpendicular to the laser propagation. The cross-section observations were carried out on the $\mathrm{X}-\mathrm{Z}$ plane perpendicular to the scanning direction after the polishing and the subsequent wet etching of the polished sample surface.

The polarization-dependent self-organized nanostructures are obviously aligned perpendicular to the writing laser polarization direction [Fig. 10(c), (d)]. Furthermore, the periodic nanostructures were also observed along the direction of laser propagation [Fig. 10(a), (b)]. This period correspond to the wavelength within the sample $(800 \mathrm{~nm} / 1.45 \sim 550 \mathrm{~nm})$. From this result, the nanostructures along the laser propagation direction are probably formed by the interference between the two photon vectors and the two electron plasma density waves. Furthermore, in the case of the focusing by high NA objective lens, the polarization-dependent periodic nanostructures were formed at the region above and below the focal spot $(\sim 15 \mu \mathrm{m})$. In the case of the high NA, the periodic nanostructure could not be observed in the region of filamentation. Therefore, the present technique will be useful in the fabrication of the polarization-dependent threedimensional optical memory devices with high storage density.

We suggest that once a high free electron density is produced by multiphoton ionization, the material has 

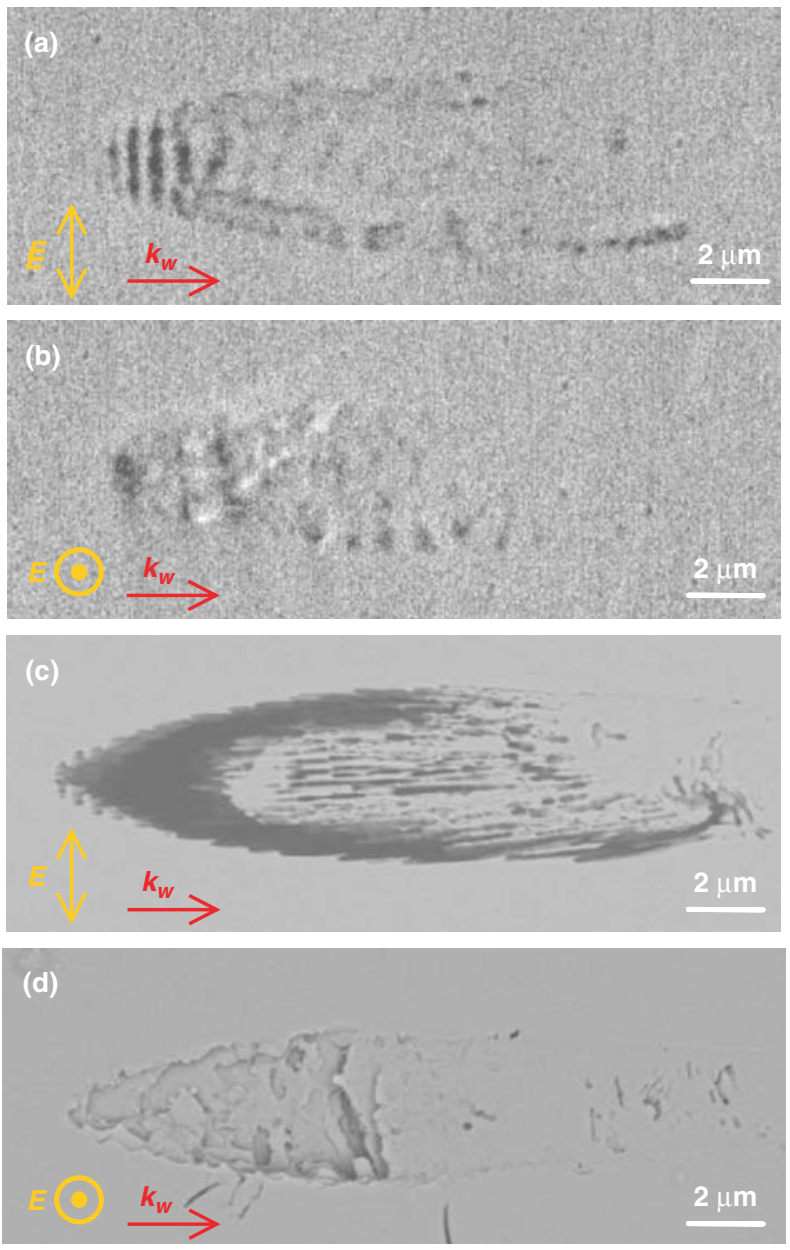

Fig. 10. Cross-sectional observation of the periodic nanostructures induced by the femtosecond single laser beam with the different linear polarization directions on the polished sample surface (a), (b) and subsequently etched surface by the hydrofluoric acid (c), (d). $K_{w}$ and $E$ show the laser propagating and polarization direction, respectively.

the properties of plasma and will absorb the laser energy via one-photon absorption mechanism of inverse Bremsstrahlung (Joule) heating. The light absorption in the electron plasma will excite bulk electron plasma density waves. These are longitudinal waves with the electric field component parallel to the direction of propagation. Such electron plasma wave could couple with the incident light wave only if it propagates in the plane of light polarization. Initial coupling is produced by inhomogeneities induced by electrons moving in the plane of light polarization. The coupling is increased by a periodic structure created via a pattern of interference between the incident light field and the electric field of the bulk electron plasma wave, resulting in the periodic modulation of the electron plasma concentration and the structural changes in glass. A positive gain coefficient for the plasma wave will lead to an exponential growth of the periodic structures oriented perpendicular to the light polarization, which becomes frozen within the material. Calculations of the period of the grating based on the momentum conservation condition and energy conservation condition show that the grating period would increase with the pulse energy. The observed increase of the grating period with the pulse energy is in agreement with this theoretical prediction.

The proposed mechanism of periodic nanostructure formation is possible to give the following explanation of the observed formation of stripe-like regions with low oxygen concentration. The plasma electrons are created in the process of breaking of $\mathrm{Si}-\mathrm{O}-\mathrm{Si}$ bonds via multi-photon absorption of light which is accompanied by the generation of a $\mathrm{Si}-\mathrm{Si}$ bonds, non-bridging oxygen-hole centers (NBOHC, $\equiv \mathrm{Si}-\mathrm{O}-)$ and interstitial oxygen atoms $\left(\mathrm{O}_{i}\right)$. Such oxygen atoms are mobile and can diffuse from the regions of high concentration. Negatively charged oxygen ions can be also repelled from the regions of high electron concentration. The photoluminescence and electron spin resonance spectra confirmed that the presence of non-bridging oxygen defects and $\mathrm{E}^{\prime}$ centers in the irradiated samples. The small thickness of these regions, compared to the period of the grating, could be explained by a highly nonlinear dependence of the structural changes on the electron concentration. Major changes in composition take place after the attainment of thermal equilibrium, involving formation and decay of defect states, such as oxygen vacancies. Detailed mechanism of the structural changes responsible for the nano-grating formation is under investigation.

\section{CONCLUSION}

We have observed various femtosecond laser-induced nanostructures in transparent materials. We demonstrated the possibility of space-selective control of refractive index and valence state of active ions (rare-earth) for the threedimensional rewriteable optical memory with ultrahigh storage density. We have also observed single femtosecond laser beam induced polarization-dependent nanostructures inside glasses. The mechanism of the nanograting formation is suggested to be due to the interaction between the coherent field of the femtosecond laser and the laserinduced plasma with matter. Recently, we also have observed formation of periodical nano-hole array along the propagation direction of the femtosecond laser beam and other interesting phenomena. We are convinced that femtosecond laser will open new possibilities in not only optical data storage, but also micro-optics, material sciences, physics, chemistry, and bioscience fields.

Acknowledgments: This work was partially supported by Japanese Ministry of Education, Culture, Sports, Science, and Technology (MEXT), and by the Mitsubishi Foundation. They are the computational materials science unit in Kyoto University, the Grant-in-Aid for Scientific Research on Priority Areas (No. 751), the Grant-in Aid for Young Scientists (B), and the 21st century COE program. 


\section{References and Notes}

1. N. Bityurin, B. Luk'yanchuk, M. Hong, and T. Chong, Opt. Lett. 29, 2055 (2004).

2. H. Misawa, Japan Patent 08-220688 (1996).

3. M. Watanabe, H.-B. Sun, S. Juodkazis, T. Takahashi, S. Matsuo, Y. Suzuki, J. Nishii, and H. Misawa, Jpn. J. Appl. Phys. 37, L1527 (1998).

4. E. N. Glezer, M. Milosavljevic, L. Huang, R. J. Finlay, T.-H. Her, J. P. Callan, and E. Mazur, Opt. Lett. 21, 2023 (1996).

5. P. P. Pronko, S. K. Dutta, J. Squier, J. V. Rudd, D. Du, and G. Mourou, Opt. Commun. 114, 106 (1995).

6. J. Qiu, K. Miura, and K. Hirao, Jpn. J. Appl. Phys. 37, 2263 (1998).

7. E. N. Glezer and E. Mazur, Appl. Phys. Lett. 71, 882 (1997).

8. J. Qiu, C. Zhu, T. Nakaya, J. Si, K. Kojima, F. Ogura, and K. Hirao, Appl. Phys. Lett. 79, 3567 (2001)

9. M. H. Hong, B. Luk'yanchuk, S. M. Huang, T. S. Ong, L. H. Van, and T. C. Chong, Appl. Phys. A 79, 791 (2004).

10. K. M. Davis, K. Miura, N. Sugimoto, and K. Hirao, Opt. Lett. 21, 1729 (1996).

11. K. Miura, J. Qiu, H. Inoue, T. Mitsuyu, and K. Hirao, Appl. Phys. Lett. 71, 3329 (1997).

12. D. Homoelle, S. Wielandy, A. L. Gaeta, N. F. Borrelli, and C. Smith, Opt. Lett. 24, 1311 (1999).

13. K. Minoshima, A. M. Kowalevicz, I. Hartl, E. P. Ippen, and J. G. Fujimoto, Opt. Lett. 26, 1516 (2001).
14. H.-B. Sun, Y. Xu, S. Juodkazis, K. Sun, M. Watanabe, S. Matsuo, H. Misawa, and J. Nishii, Opt. Lett. 26, 325 (2001).

15. B. C. Stuart, M. D. Feit, A. M. Rubenchik, B. W. Shore, and M. D. Perry, Phys. Rev. Lett. 74, 2248 (1995).

16. M. Sakakura and M. Terazima, Opt. Lett. 29, 1548 (2004).

17. M. Sakakura and M. Terazima, Phys. Rev. B 71, 024113 (2005).

18. M. Terazima, Opt. Lett. 20, 25 (1995).

19. P. Martin, S. Guizard, Ph. Daguzan, G. Petite, P. D'Oliveira, P. Meynadier, and M. Perdrix, Phys. Rev. B 55, 5799 (1997).

20. J. Qiu, K. Miura, T. Suzuki, T. Mitsuyu, and K. Hirao, Appl. Phys. Lett. 74, 10 (1999).

21. K. Miura, J. Qiu, S. Fujiwara, S. Sakaguchi, and K. Hirao, Appl. Phys. Lett. 80, 2263 (2002).

22. J. Qiu, K. Miura, K. Nouchi, T. Suzuki, Y. Kondo, T. Mitsuyu, and K. Hirao, Solid State Commun. 113, 341 (2000).

23. P. G. Kazansky, H. Inouye, T. Mitsuyu, K. Miura, J. Qiu, K. Hirao, and F. Starrost, Phys. Rev. Lett. 82, 2199 (1999).

24. J. Qiu, P. G. Kazansky, J. Si, K. Miura, T. Mitsuyu, K. Hirao, and A. Gaeta, Appl. Phys. Lett. 77, 1940 (2000).

25. Y. Shimotsuma, P. G. Kazansky, J. Qiu, and K. Hirao, Phys. Rev. Lett. 91, 247405 (2003).

26. M. Born and E. Wolf, Principles of Optics, 7th (expanded) Ed., Cambridge University Press, UK (2002), p. 837.

27. J. D. Mills, P. G. Kazansky, and E. Bricchi, Appl. Phys. Lett. 81, 196 (2002).

28. Y. Shimotsuma, K. Hirao, J. Qiu, and P. G. Kazansky, Mod. Phys. Lett. B 19, 225 (2005).

Received: 13 January 2006. Accepted: 22 February 2006. 